\title{
Pour une formation inter - didactique Mathématiques - Physique des professeurs de collège et de lycée
}

\section{Didier Malafosse2}

\section{(2) OpenEdition \\ Journals \\ Édition électronique \\ URL : http://journals.openedition.org/trema/1480 \\ DOI : $10.4000 /$ trema. 1480 \\ ISSN : 2107-0997 \\ Éditeur \\ Faculté d'Éducation de l'université de Montpellier}

\section{Édition imprimée}

Date de publication : 1 janvier 2003

Pagination : 187-209

ISSN : 1167-315X

\section{Référence électronique}

Didier Malafosse2, «Pour une formation inter - didactique Mathématiques - Physique des professeurs de collège et de lycée », Tréma [En ligne], 20-21 | 2003, mis en ligne le 12 octobre 2010, consulté le 20 avril 2019. URL : http://journals.openedition.org/trema/1480 ; DOI : 10.4000/trema.1480

Ce document a été généré automatiquement le 20 avril 2019.

Trema 


\title{
Pour une formation inter - didactique Mathématiques - Physique des professeurs de collège et de lycée ${ }^{1}$
}

\author{
Didier Malafosse2
}

\section{Introduction}

1 Dans le cadre des travaux conduits par le groupe de recherche GRIMP ${ }^{3}$, nous avons pu constater que les professeurs de collèges et de lycées qui enseignent les mathématiques ou les sciences physiques manquent de repères professionnels pour penser les continuités et les ruptures entre ces deux disciplines. Pour tenter de remédier à cette incapacité, nous avons décidé de modifier, à titre expérimental, le programme de formation initiale des professeurs stagiaires de collèges et de lycées (PLC2) du site IUFM de Perpignan dans ces deux disciplines. Le dispositif innovant que nous proposons repose sur un modèle inter-didactique d'analyse des processus de conceptualisation conçu par le groupe GRIMP. Il est basé sur les notions de cadre de rationalité, de registre sémiotique et d'espace de réalité.

Dans ce rapport, nous exposons d'abord les grandes lignes ${ }^{4}$ de ce modèle, en cherchant à mettre en évidence son double intérêt, comme outil d'analyse des conceptions des professeurs stagiaires, et comme élément du corpus de connaissances à ajouter à la formation initiale des professeurs de mathématiques et de sciences physiques. Ensuite, nous faisons une critique du dispositif actuel de formation des PLC2 et nous décrivons les modalités de mise en œuvre du nouveau dispositif de formation. 


\section{Le modèle théorique : outil d'analyse des conceptions de PLC2 et élément du corpus de connaissances de leur formation}

Le modèle d'analyse des processus de conceptualisation que nous avons élaboré repose sur un pré-modèle (Malafosse, 1999) inspiré des travaux de Balacheff (1995) distinguant, dans une perspective socio-constructiviste (Piaget, 1971; Bruner, 1991), les concepts, les connaissances, et les conceptions, et tenant compte aussi de la dualité scientifique/ quotidien (Vygotski, 1934) des concepts. Dans ce modèle, les conceptions d'un individu (et en particulier celles d'un élève ou d'un professeur stagiaire) apparaissent comme le produit d'interactions avec les objets matériels de la réalité, avec les concepts scientifiques (via la médiation didactique) et avec les concepts familiers (via la médiation sociale) (Cf. fig. 1).

Les trois sources de la conceptualisation.

Figure 1

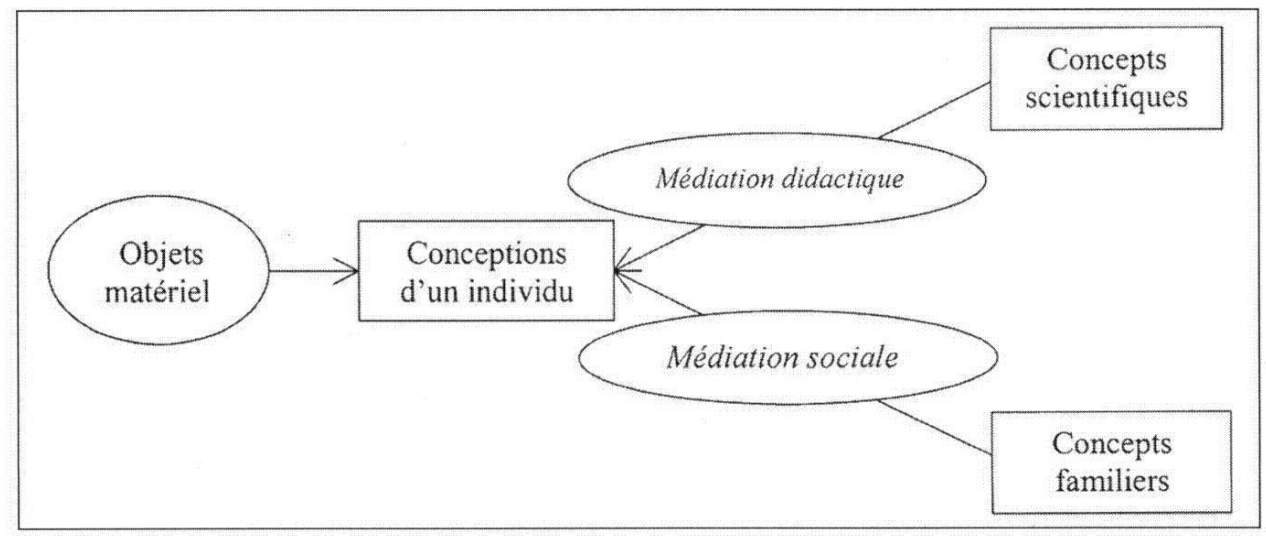

4 Cette triple origine de la conceptualisation ${ }^{5}$ nous a amenés à nous intéresser aux structures dans lesquelles s'élaborent les concepts scientifiques ou familiers d'une part, et les conceptions, d'autre part, d'où l'invention de la notion de cadre de rationalité (Lerouge, 2000c). Ensuite, pour tenir compte du statut particulier des objets réels dans la formation des conceptions, nous avons dû adjoindre à la notion de cadre de rationalité celle d'espace de réalité (Malafosse, 1999). Enfin, sensibles à l'approche sémiotique de Duval (1996), qui pose la nécessité de distinguer les productions cognitives (concepts et conceptions) des représentations qui en sont faites, nous avons fait appel à la notion de registre sémiotique (Duval, 1996). C'est sur ces trois piliers que repose notre modèle d'analyse des processus de conceptualisation.

\subsection{Registre sémiotique et congruence sémantique}

5 La notion de registre sémiotique s'impose en didactique des mathématiques et en didactique de la physique, si l'on considère comme nécessaire la distinction entre un objet conceptuel (Bunge, 1983) et les diverses représentations sémiotiques nécessaires à la fois pour le désigner et le manipuler intellectuellement. Par exemple la droite (au sens d'Euclide) peut être représentée au collège par un trait droit et cette représentation 
graphique est nécessaire à l'élève pour la conceptualiser. Mais les mathématiciens savent bien que la droite ne peut être confondue avec ce trait. En effet, l'objet mathématique sur lequel s'appuie le processus de conceptualisation n'a pas de réalité matérielle. Considérant cette distinction entre un objet conceptuel et sa représentation sémiotique comme « un point stratégique pour la compréhension des mathématiques »(Duval, 1993, p. 37), Duval a développé une approche sémiotique de la construction des connaissances en mathématique. Elle repose sur la notion de registre sémiotique qu'il définit (Duval, 1996, p. 356) comme un système de signes doté des trois «fonctions cognitives fondamentales » : la "fonction de communication", la "fonction de traitement "(c'est-à-dire la transformation de la représentation sans changer de système sémiotique), et la "fonction d'objectivation " (permettant à la fois la prise de conscience de l'existence de la représentation en tant que telle, et sa conversion dans un autre système sémiotique). Ainsi, les activités en classe de mathématiques nécessitent le recours aux registres de la langue naturelle, de l'écriture symbolique, du graphique cartésien, des figures géométriques, des tableaux de données, etc., et leur mise en œuvre coordonnée permet de réaliser les traitements cognitifs de la manière la plus économique possible.

Pour Duval, la nécessité de coordination est imposée par une autre raison beaucoup plus fondamentale liée à la construction des connaissances. En effet, "la compréhension (intégrative) d'un contenu conceptuel repose sur la coordination d'au moins deux registres de représentation, et cette coordination se manifeste par la rapidité et la spontanéité de l'activité de conversion [... mais] cette coordination est loin d'être naturelle "(Duval, 1993, p. 51). Il montre ainsi que le fait de mettre en correspondance le signe du coefficient directeur d'une droite dans le registre de l'écriture formelle des fonctions et le sens de l'inclinaison de cette droite dans le registre graphique établit une congruence entre les deux registres qui facilite l'appropriation du concept de croissance. C'est pour cette raison que Duval recommande de travailler sur la mise en congruence sémantique d'unités significatives entre divers registres pour permettre l'activité de conversion. Sans ces correspondances, il y a un cloisonnement des registres chez les élèves "qui ne reconnaissent pas le même objet à travers des représentations qui en sont données dans des systèmes sémiotiques différents: l'écriture algébrique d'une relation et sa représentation graphique »(Duval, 1993, p. 52), par exemple.

7 Les notions de registre sémiotique et de congruence sémantique présentent donc un grand intérêt dans l'analyse didactique de certaines difficultés rencontrées par les élèves et constatées par les professeurs de mathématiques. Mais elles peuvent aussi être mises en œuvre en didactique de la physique. En effet, nos travaux (Malafosse et Lerouge, 2000) ont montré que les activités cognitives en physique nécessitent aussi la mise en œuvre coordonnée de divers registres (la langue naturelle, l'écriture symbolique, le graphique cartésien, les figures géométriques, les tableaux de données, etc.), même si les règles de traitement dans ces registres dépendent de la discipline (Malafosse, 2002). Ils nous ont aussi permis de constater que des problèmes liés à l'absence de congruence sémantique existent aussi dans les activités de classe de physique.

8 Registres sémiotique et congruence sémantique étant donc des notions pertinentes en didactiques des mathématiques et de la physique, elles peuvent être considérées, déjà, comme des objets d'étude intéressants dans le cadre d'une formation professionnelle mono-didactique de professeurs stagiaires. Néanmoins, c'est dans l'approche interdidactique des processus de conceptualisation que se révèle un nouvel intérêt de la notion de registre sémiotique, lié à la particularité disciplinaire des règles de traitement à 
l'intérieur des registres sémiotiques. En effet, comme nous l'avons montré (Malafosse et Lerouge, 2000), la nature des disciplines influe sur les règles de construction des représentations sémiotiques. Par exemple, on ne peut pas réaliser, dans le registre graphique utilisé en classe de physique, certaines opérations qui sont permises dans le registre graphique utilisé en classe de mathématiques. Il y a donc rupture entre les règles de traitement de registres utilisés dans des disciplines différentes, ce qui constitue la source de difficultés d'élèves contraints de réaliser des traitements avec deux jeux de règles incompatibles, voire contradictoires. La notion de registre sémiotique présentée initialement comme un élément d'une formation didactique disciplinaire peut donc se révéler encore plus pertinente dans le cadre d'une approche inter-didactique de la formation professionnelle. Mais pour pouvoir identifier ce qui, au niveau des règles de construction des représentations sémiotiques, dépend des registres sémiotiques et ce qui dépend des disciplines, le chercheur, comme le professeur stagiaire ont besoin de deux autres notions, celle de cadre de rationalité initialement proposée par Lerouge (1992) et celle d'espace de réalité imaginée par Malafosse (1999).

\subsection{Cadre de rationalité}

9 La notion de cadre de rationalité a été construite par Lerouge (1992) pour tenir compte de la dualité familier/scientifique mise en évidence par Vygotski (1934) au sujet des processus de conceptualisation. Mise en œuvre à propos du statut de la droite au collège (Lerouge, 1992), elle a permis de mettre en évidence des contradictions dans les conceptions des élèves qui ont alors pu être analysées en termes de rupture de rationalité entre le familier des élèves et le culturel mathématique qui leur est enseigné. Dans ce cas particulier, le cadre de rationalité familier se trouve fondamentalement en rupture avec celui des mathématiques. En particulier, si le registre sémiotique des figures géométriques intervient dans les deux cadres, il ne renvoie pas du tout aux mêmes signifiés. Dans le premier cadre, la droite est identifiée au trait droit dessiné avec la règle, alors que dans le second, il s'agit d'un objet conceptuel inconnu, de nature idéelle, que le trait droit sert à conceptualiser. Mais l'intérêt de la notion de cadre de rationalité réside aussi dans son aptitude à être étendue au cas de l'interdisciplinarité en permettant une analyse en termes de continuités et de ruptures de rationalité entre le cadre familier et les divers cadres (mathématiques, physique, etc.) qui participent à la construction d'un concept en milieu scolaire.

Pour identifier les particularités liées à la rationalité du sens commun et à celles des mathématiques et de la physique, nous avons défini la notion de cadre de rationalité comme un ensemble cohérent de fonctionnement de la pensée culturelle ou personnelle, caractérisé par quatre composantes: l'ensemble des « objets conceptuels » sur lequel porte la conceptualisation, le type de processus de validation, les éléments de rationalité (règles de traitement et de validation), et enfin, les registres sémiotiques qui servent de support à la conceptualisation et à la communication. La notion de cadre de rationalité permet, alors, de distinguer des cadres culturels familiers et scientifiques, d'une part, des cadres personnels, d'autre part. On peut ainsi interpréter en termes de rupture de rationalité, aussi bien certaines difficultés d'élèves (Malafosse, 1999), que les contradictions relevés entre les conceptions des professeurs stagiaires de mathématiques et de sciences physiques à propos d'objets conceptuels et de procédures communs (Malafosse et Lerouge, 2000). Par exemple, dans ce dernier cas, les antagonismes 
constatés au niveau des conceptions des PLC2 des deux communautés résultent, pour une part, de l'histoire personnelle des individus qui, à quelques rares exceptions près, ont une culture fortement dominante. Ainsi, le professeur de mathématiques, de par sa formation universitaire, a un cadre personnel des mathématiques relativement homomorphe au cadre culturel des mathématiques. Par exemple, ces conceptions à propos du concept «droite» sont construites en référence à des connaissances institutionnellement reconnues par la communauté des mathématiciens, comme la droite d'Euclide ou celle de Hilbert. Par contre, son cadre personnel de la physique est le plus souvent fortement imprégné de sens commun (Viennot, 1996). Il en est, bien sûr, de même pour l'enseignant de physique dont le cadre personnel de la physique est relativement homomorphe au cadre culturel de la physique mais dont le cadre personnel des mathématiques est hétéromorphe au cadre culturel des mathématiques. Par exemple, sa conception de la droite est inspirée par des connaissances du sens commun. Ainsi, du fait de la dualité scientifique / familière de la conceptualisation, les connaissances des professeurs de mathématiques et de physiques à propos de la droite sont souvent opposées.

11 Les antagonismes repérés au niveau des conceptions des professeurs stagiaires ont aussi une autre origine qui doit être recherchée dans l'analyse épistémologique comparée des cadres culturels de rationalité de mathématiques et de physique. En effet, si l'on retient qu'un cadre culturel de rationalité est un système cohérent de fonctionnement de la pensée caractérisé par un ensemble de concepts, un ensemble d'éléments de rationalité et un ensemble de registres sémiotiques, force est de constater qu'il existe entre le cadre culturel des mathématiques et celui de la physique des ruptures au niveau de chacun des éléments d'identification des cadres de rationalité. Nos travaux (Malafosse et Lerouge, 2000) montrent que ces ruptures se retrouvent ensuite au niveau des connaissances à enseigner et donc des conceptions des enseignants, dans ce que nous appelons cadres culturels et cadres didactiques (Malafosse, 1999) (Cf. fig. 2). 
Figure 2

Transposition de ruptures entre les concepts scientifiques et les conceptions de l'enseignant.

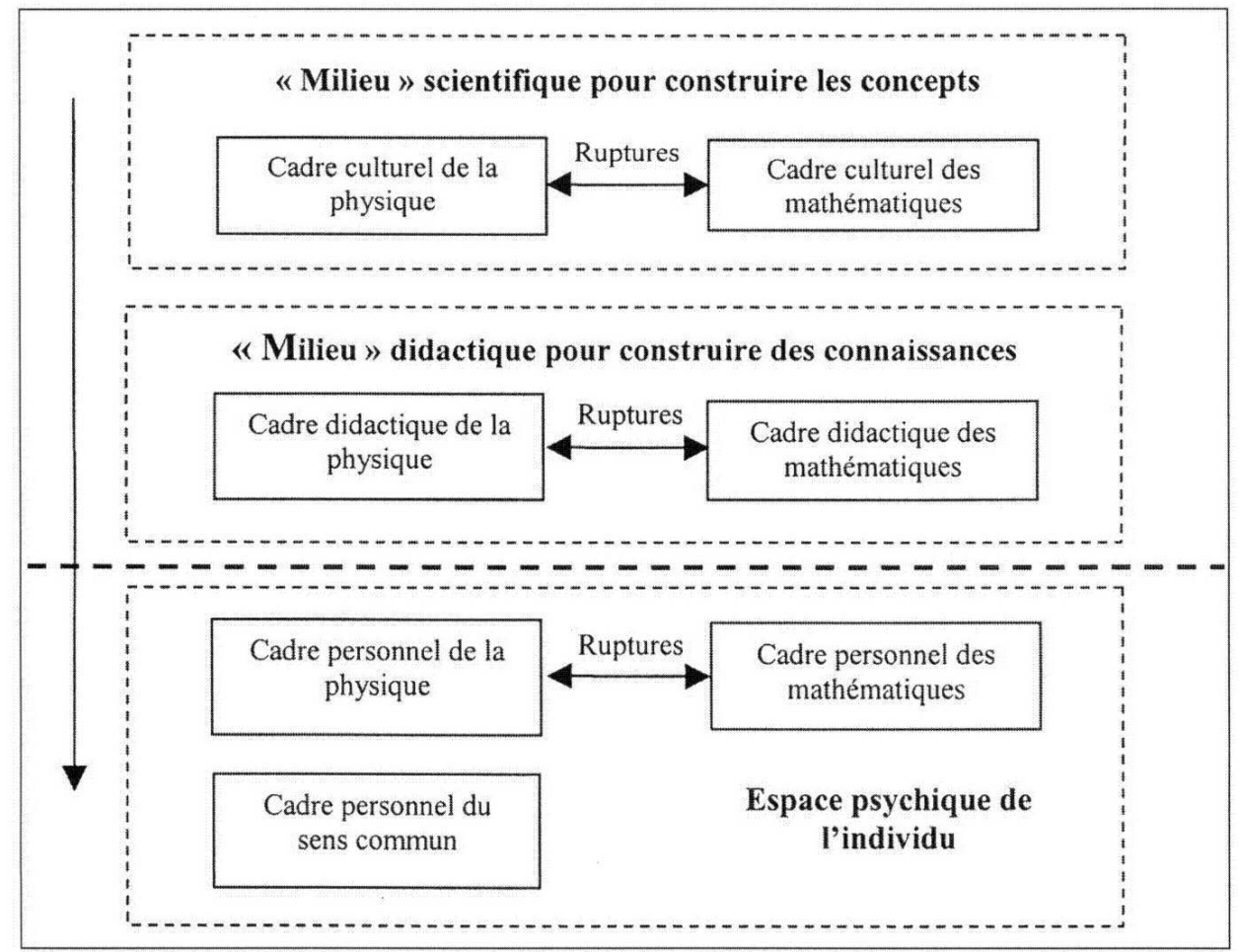

Par exemple, certains concepts appartenant à la fois au cadre culturel des mathématiques et à celui de la physique diffèrent par leurs définitions (c'est le cas pour la droite qui peut être hilbertienne en mathématique mais qui est la plupart du temps euclidienne en physique) ou par leur sens (la notion d'infini géométrique n'est pas la même dans les cadres culturels de la physique et des mathématiques). De même, les procédures de validation qui conduisent à une preuve (Balacheff, 1988) différent fortement. Enfin, nous avons montré (Malafosse, 2002) que la structure de registre sémiotique est instable dans l'opération de changement de cadre, les règles de traitement changeant suivant la nature des éléments sémiotiques mis en oeuvre. Par exemple, certaines règles de combinaison de données numériques dans un tableau de données mathématiques ne peuvent pas être appliquées à des données numériques dimensionnées comme on en trouve souvent dans les tableaux de mesures physiques.

13 En formation, la notion de cadre de rationalité présente de l'intérêt, à la fois dans les approches mono et inter-didactiques. Dans le premier cas, elle permet de modéliser les processus de conceptualisation à propos de concepts tels que la droite en mathématique au collège (Lerouge, 1992), ou la résistance électrique en physique (Malafosse, 1999). Dans cette approche, les cadres de rationalité pertinents sont le cadre personnel propre à chaque individu (dans lequel sont élaborées les conceptions inspirées à la fois par l'expérience personnelle, par la médiation de l'institution scolaire et de l'environnement social de l'individu), le cadre familier et le cadre culturel $^{6}$ dominant (celui des mathématiques ou celui de la physique). Dans l'approche inter-didactique, on doit distinguer en plus le cadre culturel de la physique de celui des mathématiques et centrer son analyse sur la recherche de ruptures de rationalité pour analyser les processus de conceptualisation. 


\subsection{Espace de réalité}

14 mathématiques et de la physique nous a amenés à réfléchir au statut des objets conceptuels manipulés dans les deux disciplines (Malafosse, Lerouge, Dusseau, 2000a). Au niveau des professeurs stagiaires, il semble qu'un consensus soit établi attribuant aux objets mathématiques un statut particulier lié à leur nature idéelle, et aux objets physiques un statut matériel. Cette distinction naïve a des conséquences au niveau didactique. Ainsi, pour Duval (1993, p. 38), "c'est seulement par le moyen des représentations sémiotiques qu'une activité sur des objets mathématiques est possible», puisque "les objets mathématiques ne sont pas directement accessibles à la perception, ou dans une expérience intuitive immédiate, comme le sont les objets communément dits 〈réels〉 ou 〈physiques〉 ».Pourtant Lerouge a montré (1992) que les difficultés de conceptualisation rencontrées par les élèves de collège sont dues, en particulier, à une rupture de nature entre l'objet « droite matérielle " constitué par le trait de crayon du cadre familier de l'élève et l'objet «droite idéelle » du cadre culturel des mathématiques. De la même manière, une analyse des processus de conceptualisation en physique montre que cette opposition entre les objets « idéels » des mathématiques et les objets dits « matériels » de la physique est dépassée. La distinction entre les « objets de la réalité » et les « objets du physicien » devient alors nécessaire. En effet, si l'on se place dans l'hypothèse épistémologique (partagée de façon implicite aussi bien par les élèves que par la majorité des enseignants de physique) d'un matérialisme réaliste (Planck, 1933), on peut affirmer que le physicien manipule des objets réels. Mais, le physicien est très rapidement amené à quitter le domaine de l'expérimentation sur les objets de la réalité pour raisonner sur des « objets » de nature très différente tels que la résistance du composant électrique, sa courbe caractéristique tension-intensité, etc. Le mot « résistor » par exemple désigne, non pas l'objet matériel « composant électrique », mais la classe des objets ayant la propriété de respecter la loi d'Ohm. La difficulté à toujours distinguer les objets réels des objets abstraits est si grande que le physicien arrive souvent à les confondre, tout au moins dans son discours. Le mot résistance n'est-il pas couramment utilisé par les enseignants (et même dans l'intitulé des programmes) pour désigner à la fois le composant électrique réel, la classe des résistors, la grandeur physique obtenue par le rapport d'une tension à une intensité, et enfin la mesure de la quantité physique repérée par la valeur numérique du coefficient $R$ d'un résistor donné?

Les professeurs stagiaires que nous avons en formation, de par leur formation universitaire, ne semblent pas conscients $d u$ fait que l'élève en phase de conceptualisation est en permanence en train de conjuguer des approches contradictoires fondées sur des visions matérialiste et idéaliste des concepts. Au delà d'une réflexion épistémologique sur la nature des objets conceptuels propre à chaque cadre de rationalité culturel, une réflexion didactique s'impose pour permettre de mettre en évidence la complexité des processus de conceptualisation. On voit donc que la notion d'espace de réalité peut aussi être introduite, dans un premier temps, par une approche monodidactique d'analyse des processus de conceptualisation que ce soit pour la formation des professeurs de sciences physiques ou pour celle des professeurs de mathématiques. 


\section{Pour une modification de la formation des PLC2 de mathématiques et de sciences physiques}

\subsection{Critique d'une formation didactique exclusivement mono- disciplinaire}

16 Notre activité de formateur en IUFM nous a permis de prendre conscience de l'importance des difficultés des professeurs stagiaires liées à une absence de repères professionnels pour penser ces continuités et ruptures entre les mathématiques et la physique. Par exemple, le professeur stagiaire de sciences physiques s'étonne lorsque les élèves n'arrivent pas à déterminer l'équation d'une droite ou la valeur d'un coefficient directeur à partir de quelques points de mesure à peu près alignés. Il ignore que l'activité attendue va à l'encontre des règles édictées en mathématiques obligeant les élèves à recourir à une démarche algébrique portant sur la droite en tant qu'objet géométrique continu. Il en va de même pour les procédures de validation d'alignement fondée sur l'interprétation graphique en physique et sur une démonstration formelle en mathématique. Les exemples de difficultés professionnelles sont nombreux et révèlent la nécessité d'une formation apportant aux stagiaires des deux disciplines des outils professionnels inter-didactiques. De plus, notre vision constructiviste de l'acquisition des connaissances impose que ces apports ne se fassent qu'après la prise de conscience par les stagiaires de leur nécessité. Or les dispositifs de formation initiale habituellement proposés en IUFM ne nous semblent permettre ni la prise de conscience par les professeurs stagiaires de la nécessité d'outils inter-didactiques, ni la mise en œuvre de ces outils dans des situations professionnelles concrètes. En effet, même s'ils tiennent compte, par l'intermédiaire d'éventuels parcours personnalisés, de l'histoire du futur professeur, les dispositifs de formation en IUFM présentent le défaut d'une centration excessive sur une approche disciplinaire de nature culturelle en première année (préparation aux concours du CAPES ou de l'Agrégation), puis de nature professionnelle en deuxième année, que ce soit au niveau des stages sur le terrain ou à celui des modules de formation didactique dispensés. Car il faut bien l'admettre, les formations en didactique reposent plus ou moins implicitement sur une approche disciplinaire héritée de l'origine même de ces didactiques et qui prévaut encore aujourd'hui. Ainsi, Chevallard, Bosch et Gascon (1997) proposent de concevoir la didactique des mathématiques comme la science de l'étude et de l'aide à l'étude des questions de mathématiques. Si une définition analogue est prise pour la didactique de la physique, comment aborder la question de la coexistence ou du partage des didactiques disciplinaires dans l'analyse de certaines activités scolaires faisant intervenir des concepts dont l'appartenance à une discipline particulière ne semble pas exclusive: par exemple, les problèmes de proportionnalité, dont Bosch (1994) reconnaît que son étude en mathématique ne se fait plus en référence à un savoir savant, mais plutôt à un ensemble de pratiques. Il faut donc admettre que les activités mathématiques et physiques nécessitent le recours à un fond commun de notions, de procédures et d'instruments. Les formations didactiques en IUFM qui restent essentiellement disciplinaires doivent donc en tenir compte et être adaptées pour que l'élève ne soit pas tiraillé entre deux triangles didactiques, celui des mathématiques et celui de la physique (Cf. fig. 3).

FIgURE 3

Approche mono-disciplinaire d'analyse didactique des processus d'enseignement /apprentissage. 


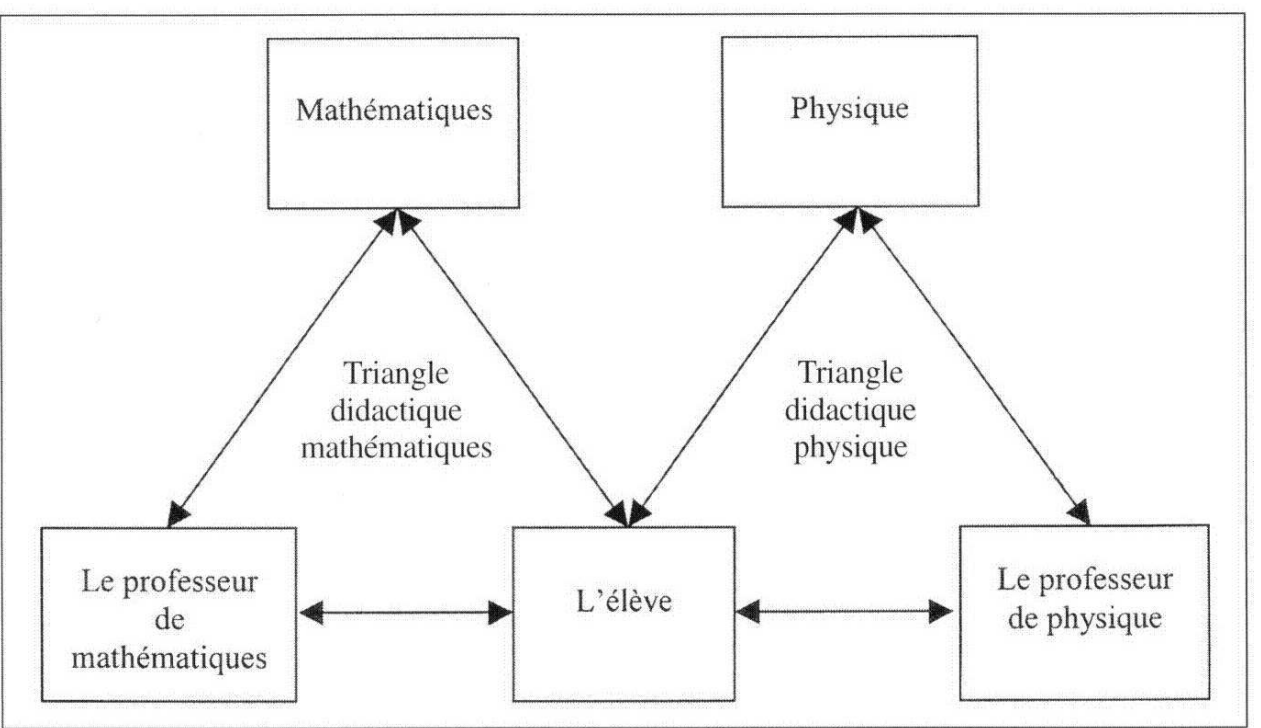

Il ne nous semble donc plus acceptable de proposer une formation initiale ayant pour effet de limiter la capacité des professeurs de mathématiques et de sciences physiques à prendre conscience des spécificités disciplinaires des savoirs savants (analyse épistémologique), des connaissances à enseigner (production de savoir à enseigner) et des outils et méthodes d'analyse de leurs pratiques professionnelles. C'est à cette fin que nous proposons de réserver pendant la deuxième année

un espace de formation professionnelle inter-didactique mathématiques-physique, à ne pas confondre avec la formation générale déjà mise en place sur les bassins de formation. L'objectif que nous lui assignons est multiple. D'une part nous attendons que les professeurs stagiaires prennent conscience de la spécificité disciplinaire de leurs conceptions à propos de concepts et de procédures qui dépassent le cadre de leurs disciplines. Nous espérons ainsi les rendre demandeurs d'outils inter-didactiques d'analyse de leurs pratiques professionnelles. Enfin, en leur proposant les moyens et les situations réelles de mises en œuvre de ces moyens, nous souhaitons développer leur goût et leur aptitude à conduire une analyse co-disciplinaire de difficultés d'apprentissage en plaçant l'élève au centre d'un triangle inter-didactique mathématiques - physique(Cf. fig. 4). 


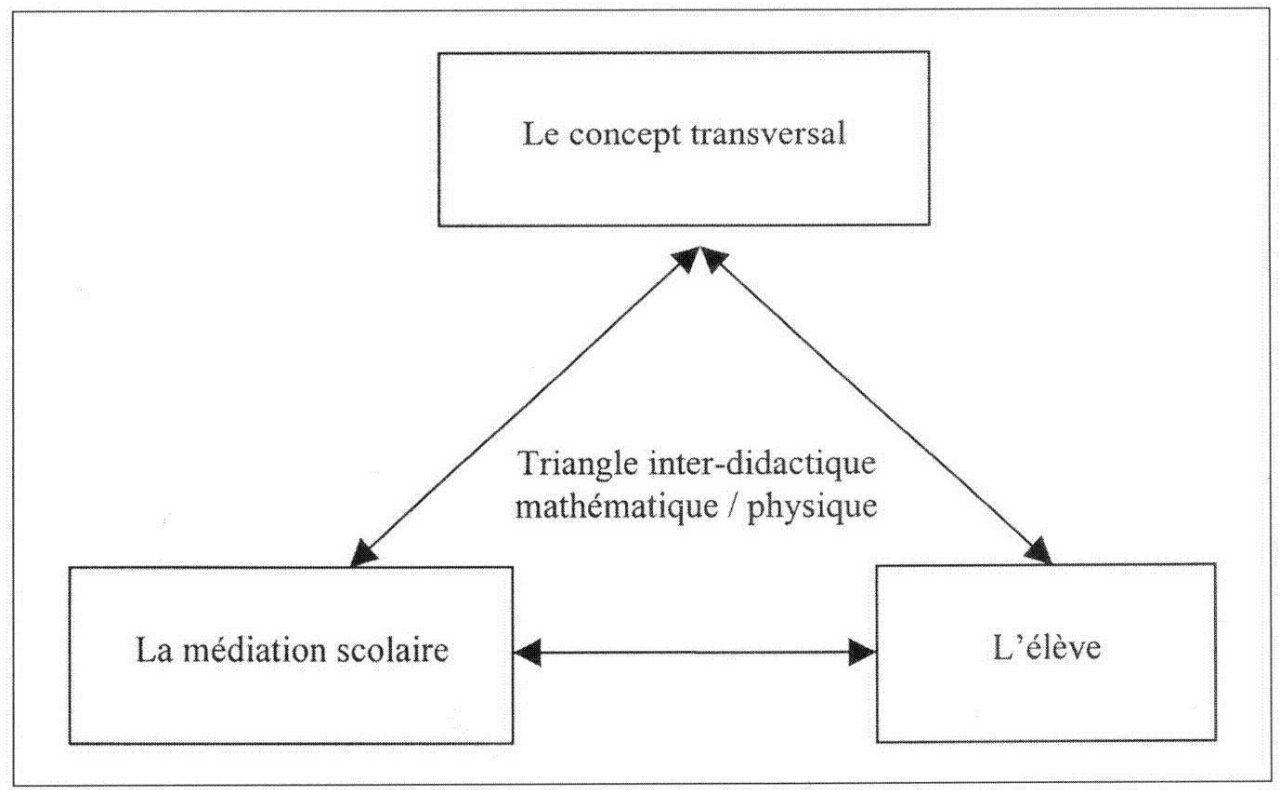

\subsection{Les grandes lignes du dispositif de formation inter-didactique}

Le dispositif que nous avons imaginé pour les professeurs stagiaires de mathématiques et de sciences physiques du site IUFM de Perpignan est constitué de six phases dont certaines ont déjà été expérimentées dans les modules de formation mono-didactique. Les premiers résultats nous ont semblé suffisamment prometteurs pour que nous envisagions de les reconduire dans un ensemble plus global.

La phase initiale consiste à opérer, dès la rentrée scolaire, un rapprochement des stagiaires des deux disciplines non plus à partir du critère disciplinaire mais plutôt à partir du critère géographique d'affectation en stage de responsabilité, pour constituer des binômes bi-disciplinaires qui seront sollicités à d'autres occasions dans le cadre des modules de formation (par exemple à l'occasion de travaux personnels demandés à la suite des séances sur les situations - problèmes, sur l'évaluation, etc.), mais aussi pour les visites formatives et, éventuellement, pour les mémoires professionnels. La deuxième phase consiste ensuite en la présentation aux stagiaires des deux disciplines, dès le début de l'année et dans le cadre de formations isolées à dominante disciplinaire, de notions issues des didactiques des mathématiques et de la physique indispensables pour constituer un fond commun nécessaire au moment du rapprochement des deux groupes de stagiaires. La troisième phase, quant à elle, se déroule dans le cadre du stage en responsabilité, à l'occasion des visites formatives : chaque stagiaire est en effet invité à participer à la préparation, à l'observation et à la critique de la visite de son collègue binômial. C'est l'occasion, pour le nouveau formé, de découvrir que l'analyse didactique dans une autre discipline nécessite des outils appartenant au fond commun élaboré, dans le cadre de sa propre formation. Plus tard dans l'année, se déroule la quatrième phase du dispositif, sous forme d'une rencontre - débat entre PLC2 des deux disciplines: c'est l'occasion de faire émerger leurs conceptions sur des objets conceptuels et des procédures de traitement et de validation communs appréhendés en tant qu'objets 
d'enseignement. Cette étape, qui sert de révélateur d'un besoin non encore satisfait par l'approche mono-didactique, est prolongée par un ensemble de visites formatives, à visée inter-didactique, associant stagiaires de mathématiques et de sciences physiques pour conduire une analyse commune de pratiques de classe (c'est la cinquième phase). Enfin, un séminaire est organisé pour structurer les divers apports théoriques inter-didactiques qui ont été distillés à l'occasion des deux phases précédentes.

\subsection{Première phase : regroupement des stagiaires par bassin de formation}

21 La phase initiale du dispositif consiste à regrouper, dès la première journée de formation, les stagiaires des deux disciplines par bassin de formation et non par discipline, pour réfléchir aux conditions de gestion de la classe qui devraient permettre d'assurer une bonne rentrée scolaire. Cette première rencontre est l'occasion, pour des stagiaires qui ont fait des études différentes, de découvrir qu'ils partagent déjà des problèmes communs : ceux liés aux aspects que nous appellerons "pédagogiques ", c'est-à-dire directement liés à la gestion de la classe, pour les distinguer des aspects "didactiques ", c'est-à-dire ceux où interviennent les trois éléments du triangle didactique. On peut, à cette occasion, demander aux stagiaires réunis en binômes bi-disciplinaires de produire une grille commune d'observation qu'ils devront utiliser, dès les premières semaines, pour aller observer, en situation de pratique de classe mais hors du cadre de la validation institutionnelle, le collègue binômial, c'est-à-dire le collègue de l'autre discipline, mais du même bassin de formation.

\subsection{Deuxième phase : présentation et mise en œuvre de notions didactiques communes}

L'objectif de formation de cette phase est de donner aux professeurs stagiaires des deux disciplines des outils communs qui leur permettront, le moment venu, d'analyser ensemble leurs conceptions à la fois au niveau de l'émergence (c'est la quatrième phase $\mathrm{du}$ dispositif) et au niveau de la mise en action sur le terrain (c'est la cinquième phase).

Pour ce faire, nous admettons qu'il existe un fond de concepts, de modèles, de théories et d'instruments communs à plusieurs didactiques, ce que Caillot et Raisky (1996) appellent le « didactique ». Nous postulons que ce fond est nécessaire aux stagiaires pour conduire des analyses mono-didactiques de pratiques professionnelles dans une discipline dont elles ne sont pas forcément issues. Nous posons aussi que, le moment venu, ce fond sera utile pour conduire des analyses de phénomènes d'enseignement/apprentissage de nature inter-didactique. C'est le cas par exemple de la notion de conception (Giordan et al, 1987), dont l'origine est plutôt à rechercher en didactique des sciences expérimentales, mais qui peut être utile pour analyser, au niveau de la mise en actes comme de la mise en mots, les stratégies des élèves en classe de mathématique. C'est aussi le cas pour les notions de situation, de système et de contrat didactiques (Brousseau, 1986, 1990) qui constituent des piliers de la didactique des mathématiques et qui, depuis quelques années, sont aussi mises en œuvre en didactique de la physique (Tsoumpelis et Gréa, 1997). C'est aussi le cas pour des notions qui dépassent la dimension disciplinaire telle que celles de transposition didactique (Chevallard, 1985), de rapport au savoir, de situationproblème, etc. 

des processus de conceptualisation en limitant, dans cette première phase, cette présentation à une dimension mono-didactique, et en la mettant en œuvre pour analyser des problèmes d'enseignement/apprentissage strictement disciplinaires. Par exemple, les notions de registre sémiotique et de congruence sémantique sont utilisées pour analyser des difficultés repérées en mathématiques à propos de l'acquisition du concept de croissance linéaire (Duval, 1988), et en sciences physiques à propos des changements de registres qui apparaissent dans la détermination graphique de lois expérimentales (Malafosse, 1999). De même, les notions de cadres de rationalité culturels et personnels sont exploitées pour étudier la rémanence de certaines conceptions. Enfin, on recourt à la notion d'espace de réalité pour interpréter les phénomènes de contagion de référent et de contagion de signifiant (Lerouge, 1993) à propos de l'intersection des droites en mathématiques ou pour interpréter, en physique, certaines difficultés liées à la détermination de la nature conductrice de certains objets (Malafosse, Lerouge et Dusseau, 2001). La présentation et la mise en œuvre de notre modèle permet ainsi aux enseignants intervenant dans les modules de didactique disciplinaire d'insister sur les aspects sémiotiques et épistémologiques de la discipline du stagiaire.

Néanmoins, comme un des objectifs finaux du dispositif est de préparer les futurs enseignants à conduire une analyse inter-didactique, il est nécessaire de profiter des nombreuses occasions rencontrées pendant le début de la formation pour développer, progressivement, une analyse épistémologique comparée des deux champs disciplinaires (par exemple comparaison des statuts de fonction mathématique et de loi physique), et une analyse sémiotique comparée (par exemple analyse comparée des règles de traitement des registres sémiotiques mis en œuvre en classes de mathématiques et de physique). Ainsi, la réflexion sur l'analyse des processus de conceptualisation développée dans la première phase de la formation pourra s'enrichir par le passage, en cours d'année de formation, de la perspective mono-didactique à la perspective inter-didactique. L'approche limitée, dans la phase de la formation, à la dualité cadre de rationalité personnel / cadre de rationalité culturel (physique ou mathématique) pourra devenir en temps voulu, trilogique en ajoutant une distinction entre cadres culturels mathématique et culturel physique. Ceci permettra alors aux professeurs stagiaires d'interpréter, en fin de formation, les difficultés constatées d'élèves en termes de ruptures de rationalité entre les deux disciplines et d'envisager des remédiations qui n'auraient pu être imaginées par une analyse exclusivement mono-disciplinaire des processus d'enseignement / apprentissage.

\subsection{Troisième phase : la participation à la visite formative du collègue binômial}

26 L'objectif des visites formatives est de fournir aux stagiaires des situations pratiques pour conduire une analyse croisée de la pratique professionnelle. Le dispositif retenu est une variante $^{6}$ de celui mis au point par une équipe co-disciplinaire de l'IUFM de Montpellier, dans le cadre du projet ESTEREL ${ }^{7}$ sur l'intégration de la formation par alternance. Il est appliqué depuis plusieurs années, avec des protocoles légèrement différents, en formation des PLC2 de mathématiques et de sciences physiques, pour la visite qui se situe au début du stage en responsabilité, mais sa gestion n'était assurée, jusqu'à aujourd'hui, que dans une perspective mono-disciplinaire.

Tréma, 20-21 | 2003 
La démarche consiste à dégager du cours d'action d'un professeur stagiaire des situations en actes (Lerouge, 2000a et 2000b), puis à développer sur ces situations un repérage professionnel théorique. La visite formative est construite autour d'une observation réalisée dans la classe d'un professeur stagiaire. Elle se déroule sur trois phases: la préparation de l'observation, l'observation proprement dite, et l'analyse de la pratique observée. Cette observation donne lieu à un travail collectif d'analyse de pratique auquel participent quatre à cinq personnes : le stagiaire accueillant qui fait la leçon, trois à quatre observateurs (des professeurs stagiaires de la même discipline et le professeur stagiaire binômial), le conseiller pédagogique et un des enseignants du stagiaire accueillant, la participation conjointe de ces deux formateurs IUFM permettant l'intégration des deux versants pratique et théorique de la formation par alternance. L'ensemble s'inscrit dans une dynamique de formation réflexive du groupe des participants, tant pour celui qui fait la leçon que pour ceux qui l'observent. La leçon observée est préparée par le professeur stagiaire sur un thème s'intégrant à la progression retenue pour la classe d'observation. Ce choix d'une construction de séance en « solitaire » a certes le défaut d'être mal dévolue aux observateurs, mais il a l'avantage de permettre d'analyser le cours d'action de l'enseignant en fonction de sa propre prévision en dehors de toute contrainte normative.

\subsubsection{La préparation de l'observation}

L'entretien qui prépare l'observation a deux objectifs : permettre la dévolution de la séance prévue aux observateurs, et répartir les tâches d'observation. Ce travail doit se faire sans déstabiliser l'enseignant qui va faire la leçon, ce qui suppose de baliser les échanges au niveau d'une prise d'information en interdisant toute discussion critique sur les choix présentés. Dans un premier temps, le professeur stagiaire présente au groupe des observateurs sa séance sur la base du document ci-suit (Cf. tableau 1).

\section{TABLEAU 1}

Document d'analyse des situations prévues et réalisées.

\begin{tabular}{|l|l|}
\hline \multicolumn{2}{|c|}{\begin{tabular}{l|} 
- Objectif de la séquence de référence \\
- Objectifs de la séance observée \\
- Types de situations prévues pour la séance
\end{tabular}} \\
\hline \multicolumn{2}{|c|}{ Situation 1 de type... } \\
\hline Prévision & Réalisation \\
\hline $\begin{array}{l}\text { - Durée } \\
\text { - Objectifs } \\
\text { - Dispositif } \\
\text { - Points à observer }\end{array}$ & - Durée \\
\hline
\end{tabular}

En bandeau initial, ce document situe la séance dans la séquence de référence, puis rend compte en partie gauche de la prévision de l'enseignant, situation par situation. En partie droite apparaît la réalisation qui sera renseignée par les observateurs. La rubrique " points à observer ", invite l'acteur à faire part de ses interrogations prévisionnelles et à présenter sa réflexion sur des choix alternatifs. Cela l'amène à relativiser ses options, et à engager lui-même les observateurs à en vérifier l'efficacité dans l'action. La séance apparait alors d'avantage comme la stabilisation nécessaire de choix professionnels à analyser, plutôt que comme un objet figé dont le concepteur supporterait mal la critique. 
Les tâches d'observation sont réparties sur la base de trois entrées que sont le fonctionnement du dispositif prévu, le jeu de l'enseignant dans ce dispositif, et enfin, l'observation de l'activité des élèves. Sur ces grands chapitres, chaque stagiaire spécifie ce qu'il envisage d'observer de manière plus particulière, et dont il rendra compte au groupe pour nourrir l'analyse qui suit la leçon. Le conseiller pédagogique et le formateur IUFM n'ont pas de tâche d'observation préalablement assignée, leur rôle consistant essentiellement à repérer les noyaux autour desquels ils engageront la démarche d'analyse.

\subsubsection{L'observation} $\mathrm{du}$ document que nous venons de décrire. En fonction de la problématique professionnelle développée par l'enseignant lors de la présentation de sa prévision, leur tâche consiste à repérer les écarts entre le prévu et le réalisé et à analyser ces écarts à la fois du point de vue didactique et pédagogique. Parallèlement à cette observation du dispositif, d'autres observateurs se focalisent sur le comportement local et général de l'enseignant soit sur la base de la grille d'observation élaborée en début d'année avec son collègue binômial, soit à partir de toute autre grille plus élaborée ${ }^{(8)}$. Enfin, l'enseignant indique aux observateurs deux ou trois élèves dont il pense intéressant d'observer le cours d'action pendant la séance. Chaque élève désigné est pris en charge par un observateur qui peut se positionner à proximité, sa tâche consistant à réaliser un relevé précis de son activité cognitive et de son comportement. Pratiquement, l'observation se fait sur la base d'un tableau identifiant les phases stabilisées du cours d'action de l'élève, au regard desquelles l'observateur propose des hypothèses explicatives à caractère didactique ou pédagogique.

\subsubsection{L'analyse de l'observation}

31 Elle se déroule sur la base d'un protocole en cycles "action-modélisation » étayés par l'ensemble des données d'observation que nous venons de mentionner. Par cycle action modélisation, nous désignons un temps d'analyse de la pratique constitué de deux phases : une phase d'analyse intrinsèque de l'action visant à expliciter l'organisation en acte de l'enseignant suivie d'une phase d'analyse extrinsèque (théorisation) de cette organisation visant à développer un repérage professionnel transférable. Sous peine de s'inhiber mutuellement, ces deux phases doivent être clairement balisées par les animateurs sur des temps différents de la démarche d'analyse. Tout particulièrement, il est essentiel de faire en sorte que les logiques d'action en situation puissent être explicitées. Cela interdit toute validation ou modélisation prématurées qui ne peuvent qu'occulter l'approche énactée. Dans ce contexte, le rôle des animateurs est essentiel : ils doivent accompagner les échanges sur l'analyse intrinsèque de l'action jusqu'à ce qu'ils jugent qu'une structure didactique ou pédagogique en acte soit suffisamment cernée pour pouvoir être analysée de manière extrinsèque. L'objectif de la phase de modélisation est alors de dégager des repères professionnels transférables. La consigne est inversée : il ne s'agit plus d'entrer dans l'intrinsèque de l'action, mais de réagir par rapport à des «théories » personnelles que chacun applique à l'action pour en tenter une explication. Pour éviter d'inhiber cette démarche par un enfermement polémique avec l'acteur, on lui demande de ne pas intervenir, en lui assurant un temps de réaction en fin du cycle 
d'analyse. Cette phase est un moment clé de l'étayage inter-didactique du transfert professionnel dans le sens où elle réalise l'intégration des connaissances théoriques de professeurs stagiaires de sciences physiques et de mathématiques à la pratique observée et explicitée. Néanmoins, une mise en place institutionnelle des éléments théoriques d'analyse inter-didactique des processus de conceptualisation est nécessaire. Elle est réalisée dans la sixième et dernière phase du dispositif, sous forme d'une conférence coassurée par le formateur de didactique des mathématiques et par celui de didactique de la physique.

32 Du point de vue qui nous intéresse, on peut considérer que cette visite formative, à laquelle le stagiaire binômial participe en tant qu'«invité » n'est qu'une étape de familiarisation à l'analyse mono-didactique d'une autre discipline. En réalité, au delà du rapprochement des personnes de deux disciplines différentes, cette phase est l'occasion, pour le nouveau formé, de découvrir que l'analyse didactique dans une autre discipline nécessite des outils appartenant au fond commun élaboré, dans le cadre de sa propre formation.

\subsection{Quatrième phase : rencontre - débat à propos d'objets conceptuels communs}

33 Ce dispositif, dont Legrand (1988) a montré l'intérêt, a déjà été expérimenté dans le cadre de nos travaux de recherche pour faire émerger les conceptions des PLC2 de mathématiques et de sciences physiques à propos d'objets conceptuels et de procédure de traitement et de validation intervenant dans les deux disciplines. Il a été décrit et ses résultats ont été publiés (Malafosse et Lerouge, 2000). Nous nous proposons de le reconduire mais avec une visée formative et non plus de recherche, et cette reconduction nécessite une importante transposition. L'objectif est de faire prendre conscience aux stagiaires de la spécificité culturelle de leurs conceptions. Les fondements de la mise en œuvre de la stratégie retenue repose sur une triple hypothèse. La première, de nature épistémologique, pose l'existence d'un rapport différent à l'espace de réalité dans les deux disciplines, ce qui se traduit par des ruptures tant au niveau des concepts qu'au niveau des procédures de raisonnement et de validation. La seconde, de nature sémiotique, concerne plus particulièrement le statut des représentations graphiques aussi bien dans leur fonction de représentation que dans celle de traitement. Enfin, la dernière hypothèse pose que le professeur stagiaire a des conceptions sur les objets et procédures à enseigner qui présentent avec les objets et procédures du savoir savant un homomorphisme conservant les ruptures culturelles inter-disciplinaires. Pour mettre en évidence des écarts de conceptions entre les stagiaires des deux communautés, nous avons décidé de retenir le champ conceptuel de la proportionnalité qui est abordé en fin de collège dans les deux disciplines et qui nous parait particulièrement marqué par ces ruptures. Le dispositif consiste alors à organiser, pendant une demi-journée, une rencontre entre une dizaine de PLC2 de mathématiques et autant de PLC2 de sciences physiques sous forme d'un débat à propos des divers éléments graphiques mis en œuvre dans le traitement des graphes cartésiens. La situation d'entrée dans l'activité consiste en un questionnaire relatif à la représentation cartésienne souvent utilisée par les élèves en fin de troisième, tant dans le cadre scolaire des mathématiques (représentation des fonctions) que dans celui de la physique (caractéristiques des résistors, etc.). Un débat est ensuite organisée autour de quelques questions-clés du dit questionnaire. 
34 Nos expériences précédentes de ce dispositif nous ont montré que les professeurs stagiaires des deux communautés sont surpris de découvrir que leurs approches épistémologiques et didactiques sont très différentes. C'est par exemple le cas à propos du statut de la droite, qui, pour les mathématiciens, " existe sans être représentée ", alors qu'« elle n'est qu'un outil de travail » sans existence propre pour les physiciens. Ces ruptures au niveau du statut des objets trouvent un écho au niveau des conceptions des enseignants des deux communautés sur les compétences scolaires espérées. Ainsi, les PLC2 attendent qu'en classe de mathématique, on pense d'abord la droite puis qu'on la visualise et que par déduction, on s'intéresse à ses points, alors qu'en classe de sciences physiques, ils attendent qu' "on parte d'un nuage de points pour modéliser» par induction. Bref, ces débats permettent de faire un inventaire des différences de conceptions des membres des deux communautés de stagiaires à propos des éléments sémiotiques intervenant dans les graphiques et à propos de leur utilisation en classe. Par cette prise de conscience, la confrontation devient un moyen de déstabiliser les professeurs stagiaires sur l'exclusivité de validité de leurs conceptions et de leurs objectifs professionnels et sur l'illusion de transparence de l'opération de changement de cadre de rationalité. En particulier, ils découvrent que la structure de registre sémiotique n'est pas stable pendant l'opération de changement de cadre de rationalité.

Par ailleurs, ils réalisent qu'il existe des ruptures entre leur cadre personnel et le cadre culturel de la discipline qu'ils n'enseignent pas. Enfin, le débat est un moyen de mettre en évidence la nécessité de disposer de repères professionnels inter-didactiques pour ne pas tomber dans l'illusion de la transparence du changement de cadre de rationalité. Ceci permet la mise en œuvre de ces repères professionnels inter-didactiques au cours d'une approche finalisée sous forme d'observation et d'analyse communes de séances de cours : c'est le dispositif des visites formatives à visée inter-didactique.

\subsection{Cinquième phase : les visites formatives à visée interdidactique}

36 Cette phase qui se déroule au mois de mai, juste après les étapes de validation de la formation par les instances universitaires et administratives, repose sur l'extrapolation à visée inter-didactique du dispositif des visites formatives déjà mis en œuvre en début d'année dans le cadre institutionnel de formation - validation. Notre projet est de transposer ce dispositif déjà bien rodé et connu des stagiaires, pour lui conférer une pertinence inter-didactique. Le but est d'intégrer les aspects didactiques (liés à deux disciplines différentes) et pédagogiques de la formation, en utilisant l'analyse de pratiques spontanées observées sur le terrain. La mise en œuvre de visites formatives dans le cadre d'une formation inter-didactique permet ainsi une approche à la fois codisciplinaire et multi-référencée dans laquelle chaque champ théorique donne un regard spécifique sur la pratique, sans chercher à intégrer ces divers champs dans une matrice explicative unique. La visite formative devient alors un dispositif où se croisent des analyses didactiques habituellement isolées.

Bien que son objectif prioritaire soit de nature inter-didactique, le dispositif retenu ressemble beaucoup à celui des visites formatives institutionnelles, car il est encore structuré autour des trois temps : préparation, observation, analyse. 


\section{La préparation de l'observation} formateurs responsables de la formation des PLC2 des deux disciplines (un de mathématiques et un de sciences physiques), en partant d'exemples concrets relevés à l'occasion des visites formatives (phase précédente). C'est l'occasion de faire participer les stagiaires, tout en les contraignant à mettre en œuvre dans une perspective interdisciplinaire, les outils didactiques présentés pendant toute l'année.

Dans un deuxième temps, une synthèse est réalisée par un des deux formateurs pour replacer le modèle d'analyse des processus de conceptualisation dans une perspective à la fois intégratrice et différentiatrice, en mettant en exergue l'intérêt de connaître et de savoir utiliser des concepts didactiques élaborés dans différentes disciplines, mais en mettant en évidence les différences de rationalité de ces disciplines. 


\section{9. Évaluation}

La fin du séminaire est consacrée, à l'aide d'un questionnaire distribué, à une évaluation par les stagiaires du dispositif expérimental. Leur avis doit nous aider à améliorer les aspects matériels, méthodologiques, et théoriques de ce module innovant de formation. Il est en effet impossible de ce prononcer, dès le départ, à la fois sur la pertinence de l'analyse a priori sur laquelle il est fondé, et sur la pertinence des choix de situations didactiques retenues. Comme tout dispositif expérimental de formation, ce n'est qu'après analyse a posteriori qu'un bilan sérieux peut être fait. Néanmoins l'accueil réservé par les stagiaires à certaines phases du dispositif en pré-expérimentation et à la présentation du projet nous semble dès à présent très favorable.

\section{3. État d'avancement du projet}

La stratégie initialement retenue pour la mise en œuvre de notre projet innovant de formation reposait sur un calendrier flou mais sur une chronologie très précise: 1) finalisation $\mathrm{du}$ modèle théorique, 2) conception et stabilisation d'un dispositif expérimental, 3) pré-expérimentation de certains éléments du dispositif, 4) formation des formateurs, 5) mise en œuvre globale, 6) évaluation. Après avoir modifié plusieurs fois sur le papier le protocole de mise en œuvre du dispositif, nous sommes arrivés aujourd'hui à la phase de formation des formateurs et pensons être en mesure de mettre en place dans toute ses dimensions le dispositif innovant avec la prochaine promotion de professeurs stagiaires de lycées et collèges. C'est dans cette perspective que nous réfléchissons en ce moment aux calendriers des professeurs stagiaires de mathématiques et de sciences physiques affectés sur le site de Perpignan pour la rentrée prochaine. En effet, ce n'est qu'appliqué dans son intégralité que ce dispositif prendra la dimension inter-didactique qui lui donne sens. Il est donc indispensable de poursuivre, pendant l'année universitaire à venir, notre démarche expérimentale, mais aussi de coupler la formation des stagiaires avec la formation de quelques formateurs supplémentaires, dans l'éventualité de la généralisation du dispositif à tous les PLC2 de l'académie. 


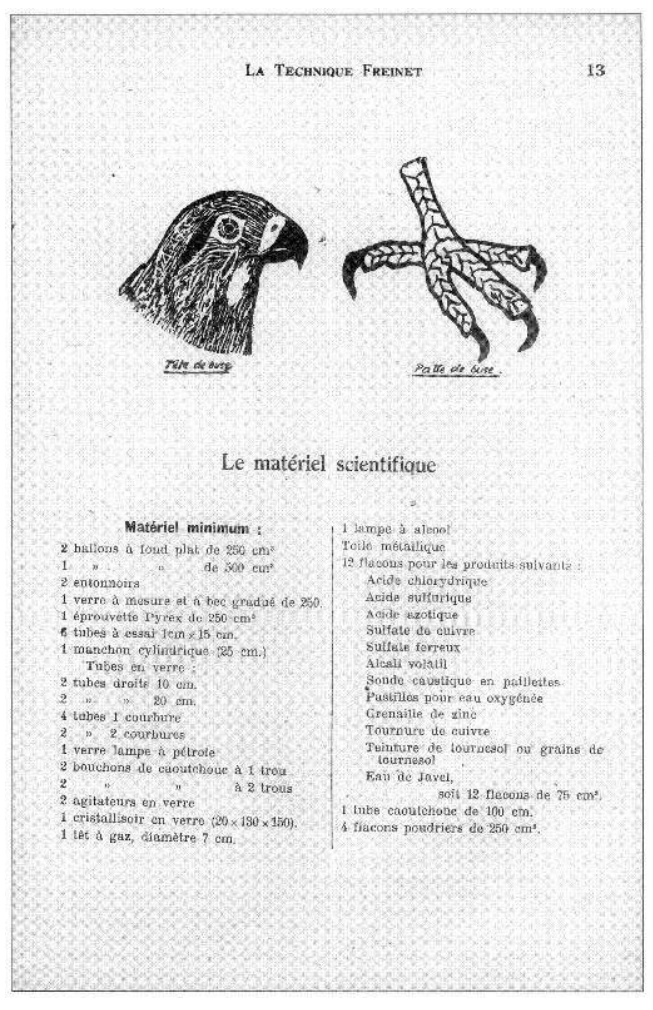

\section{BIBLIOGRAPHIE}

BALACHEFF N. : Une étude des processus de preuve en mathématique chez des élèves de collège. Thèse d'état, Université J. Fourier, Grenoble, 1988.

BALACHEFF N. : «Conception, propriété du système sujet / milieu », in Actes de la VII École d'été de didactique des mathématiques, 1995, pp. 215-229.

BOSCH M. : «Les instruments du travail mathématique : le cas de la proportionnalité », in ARTIGUE M. et al. (Éds.), Vingt ans de didactique des mathématiques en France. Grenoble, La Pensée Sauvage, 1994.

BROUSSEAU G. : « Fondements et méthodes de la didactique des mathématiques », in Recherche en Didactique des Mathématiques, Vol. 7, º 2, 1986.

BROUSSEAU G. : « Le contrat didactique : le milieu », in Recherches en Didactique des Mathématiques. Vol. 9, N 3, 1990, pp. 309-336.

BRUNER J. .... Car la culture donne forme à l'esprit. Paris, Eshel, 1991.

BUNGE M. : Épistémologie. Paris, Éditions Maloine (Collection : Recherches Interdisciplinaires), 1983.

CAILLOT M. et RAISKY C : Au-delà des didactiques : le didactique. Bruxelles (Belgique), DeBœk, 1996, pp. 9-15. 
CHEVALLARD Y. : La transposition didactique. Grenoble, La Pensée sauvage, 1985.

CHEVALLARD Y., BOSCH M. et GASCON J. : Estudiar matemáticas. El eslabón perdido entre enseñanza y aprendizaje. Barcelona (Espagne), ICE-Horsoni, 1997.

DUVAL R. : « Registres de représentation sémiotique et fonctionnement cognitif de la pensée », in Annales de Didactique et de Sciences Cognitive de l'IREM de Strasbourg, 5, 1993, pp. 37-65. DUVAL R. : «Quel cognitif retenir en didactique des mathématiques?», in Recherche en Didactique des Mathématiques, Vol. 16, N 3, 1996, pp. 349-382.

GIORDAN A. et al. : L'élève et/ou les connaissances scientifiques. Berne (Suisse), Peter Lang, 1987 ( 2 édition).

LEGRAND M. : « Genèse et étude sommaire d'une situation co-didactique : le débat scientifique en situation d'enseignement ", in Actes du premier colloque franco-allemand de didactique des mathématiques. Grenoble, La pensée sauvage, 1988, pp. 53-66.

LEROUGE A. : Représentation cartésienne, rationalité mathématique et rationalité du quotidien chez des élèves de collège. Thèse de doctorat, Université Montpellier II, 1992.

LEROUGE A. : «Contagion de signifiant et contagion de référence sur la conceptualisation mathématique de l'intersection de deux droites ", in Les sciences de l'éducation pour l'ère nouvelle, $\mathrm{N}^{\circ} 1-3,1993$, pp. 119-135.

LEROUGE A. : «L'analyse dialogique de l'action située de l'enseignant : principes et mise en œuvre dans la formation de professeurs stagiaires en IUFM », in Revue Française de Pédagogie, 2000a.

LEROUGE A. : « La visite de classe formative : un noyau d'intégration des formations didactiques et pédagogiques dispensées à l'IUFM », in Revue Recherche et formation, $2000 \mathrm{~b}$.

LEROUGE A. : « La notion de cadre de rationalité à propos de la droite au collège (à paraître) », in Recherche en Didactique des Mathématiques, Vol. 20, N², 2000c, pp. 171-208.

MALAFOSSE D. : Contribution à l'analyse et à la modélisation des processus de conceptualisation en inter-didactique des mathématiques et de la physique : exemple de la loi d'Ohm. Thèse de Doctorat, Université Montpellier II, 1999.

MALAFOSSE D. : « Pertinence des notions de cadre de rationalité et de registre sémiotique pour l'analyse des processus de conceptualisation en didactique de la physique (à paraître) », in Recherche en Didactique des Mathématiques, Vol. 22, № 1, 2002, pp. 31-76.

MALAFOSSE D. et LEROUGE A. : «Ruptures et continuités entre physique et mathématique dans l'enseignement de la notion de caractéristiques des dipôles électriques linéaires ", in Aster, $\mathrm{N}^{\circ} 30$, 2000, pp. 65-85.

MALAFOSSE D. et LEROUGE A. : «D'une recherche inter-didactique mathématiques/physique à un projet de formation initiale des professeurs de collèges et lycées ", in Aster, $\mathrm{N}^{\circ} 32,2001$, pp. 123-145.

MALAFOSSE D., LEROUGE A. et DUSSEAU J.-M. : « Étude en inter-didactique des mathématiques et de la physique de l'acquisition de la loi d'Ohm au collège : I. Espace de réalité ", in Didaskalia, $\mathrm{N}^{\circ}$ 16. 2000a, pp. 81-106.

MALAFOSSE D., LEROUGE A. et DUSSEAU J.-M. : « Notion de registre et de cadre de rationalité en inter-didactique des mathématiques et de la physique ", in Tréma, N 18, 2000b, pp. 49-60. 
MALAFOSSE D., LEROUGE A. et DUSSEAU J.-M. : « Étude en inter-didactique des mathématiques et de la physique de l'acquisition de la loi d'Ohm au collège : II. Changement de cadres de rationalité », in Didaskalia, Nº 18, 2001, pp. 61-98.

PIAGET J. : La psychologie de l'enfant. Paris, P.U.F. (collection : Que sais-je ?), 1971.

PLANCK M. : L'image du monde dans la physique contemporaine. Paris, Gonthier, 1933, pp. 5-7.

POSTIC M. : Observation et formation des enseignants. Paris, P.U.F., 1977.

TSOUMPELIS L. et GREA J. : « Essai d'application de la théorie des situations en sciences physiques ", in Recherches en Didactique des Mathématiques, Vol. 15, N" 2, 1995, pp. 63-108.

VIENNOT L. : Raisonner en physique : la part du sens commun. Bruxelles (Belgique), DeBœk, 1996. VYGOTSKI L.S. : Thought and Language. Cambridge (Mass., USA), MIT Press, 1962 (1 ${ }^{\text {re }}$ éd. : 1934).

\section{NOTES}

1. Ce rapport de synthèse de projet innovant a été rédigé en empruntant et en réactualisant une partie de l'article publié par Didier Malafosse et Alain Lerouge dans le numéro 32 de la revue Aster (Malafosse et Lerouge, 2001).

2. Professeur agrégé de physique au site IUFM de Perpignan, diplômé d'études approfondies de "physique des hautes énergies" et de "didactique des disciplines scientifiques", Docteur en " didactique des disciplines scientifiques».

3. Le groupe GRIMP (Groupe de Recherche en Inter-didactique des Mathématiques et de la Physique), de l'IUFM de l'Académie de Montpellier, fait partie de l'Équipe ERES (Equipe de Recherche sur l'Enseignement des Sciences, EA 730) de l'Université Montpellier II. Il est constitué d'enseignants et d'enseignants-chercheurs de l'IUFM exerçant une part de leur service en formation initiale des professeurs stagiaires de mathématiques et de sciences physiques.

4. Le lecteur intéressé trouvera une description plus détaillée de ce modèle et de ses composantes dans le numéro 18 de la revue Tréma (Malafosse, Lerouge et Dusseau, 2000b).

5. Nous préférerions le terme de conceptionalisation plutôt que celui de conceptualisation, dans la mesure où l'on s'intéresse à la construction des conceptions plutôt qu'à celle des concepts.

6. Il s'agit en fait de cadres de rationalité didactiques dans lesquels les objets conceptuels, les règles de traitement, les théories, etc. sont obtenus par transposition didactique des savoirs savants des cadres culturels scientifiques (Malafosse, 1999).

7. Etude Systémique des TERritoires Educatifs Locaux : équipe de recherche co-disciplinaire de l'IUFM de Montpellier.

8. Par exemple, la grille proposée par Postic dans « Observation et formation des enseignants » (1977, p. 244-245) peut constituer une bonne base de travail pour le stagiaire dans sa tâche de construction d'une grille d'évaluation de la performance en situation de pratique de classe. Sur le fond et dans la forme, ce document peut être librement personnalisé en modifiant tout ou partie des items du modèle, de telle sorte qu'il propose aux observateurs un document dont il accepte a priori l'utilisation pendant la visite qui le concerne. 


\section{RÉSUMÉS}

Pour aider les professeurs de collèges et de lycées qui enseignent les mathématiques ou les sciences physiques à mieux appréhender les continuités et les ruptures qui existent entre ces deux disciplines, nous avons modifié le programme de leur formation initiale. Cet article présente le dispositif innovant que nous avons mis en place à l'IUFM de l'académie de Montpellier.

Il repose sur un modèle inter - didactique d'analyse des processus de conceptualisation basé sur les notions de cadre de rationalité, de registre sémiotique et d'espace de réalité. Nous présentons ici le modèle et ses trois composantes, puis à partir de la critique d'une formation didactique mono - disciplinaire, nous développons, en en justifiant l'intérêt, les divers éléments du nouveau dispositif de formation.

To help student teachers of mathematics and physics reflect on both the break and continuity points that exist between these two disciplines, we have modified their initial training program. This article presents the new training methods put in place at the IUFM of Montpellier. Firstly we will present the new methods, based on a theoretical model, followed by an examination of the traditional methods of training secondary school teachers. Lastly we will describe the different steps of putting in place the new methods which, we believe, will solve the interdisciplinary challenges we face today.

\section{INDEX}

Mots-clés : formation, innovation, inter-didactique, mathématiques, professeur stagiaire, sciences physiques

Keywords : inter-didactic, mathematics, new method, physics, student teacher, training program

\section{AUTEUR}

\section{DIDIER MALAFOSSE2}

Professeur agrégé, IUFM de Perpignan 